\title{
Intraocular pressure measured by dynamic contour tonometer and ocular response analyzer in normal tension glaucoma
}

\author{
Zilin Chen • Guihua Xu
}

Received: 24 September 2009 / Accepted: 20 October 2009/Published online: 24 November 2009

(C) Springer-Verlag 2009

\section{Dear Editor,}

We read with interest the article by Morita $\mathrm{T}$ et al. on intraocular pressure (IOP) measured by dynamic contour tonometer (DCT) and ocular response analyzer (ORA) in normal tension glaucoma (NTG) [1]. In their paper, the authors presented that corneal-compensated IOP (IOPcc) measured by ORA was significantly greater than GAT-IOP, DCT-IOP and Goldmann-correlated IOP (IOPg) in NTG eyes, but not in normal eyes, which suggested the possibility of IOP values being underestimated. We would like to raise a few points with regard to this article that may help to broaden the discussion.

It is well-recognized that IOP measurement can be affected by central corneal thickness (CCT) and the biomechanical properties of the cornea, such as corneal hysteresis $(\mathrm{CH})$, corneal resistant factor (CRF) and so on [2]. Corneal hysteresis may vary from different subjects. Sunil et al. reported that corneal hysteresis was lowest in NTG eyes when comparing with POAG and OHT eyes [3]. On the other hand, Ang GS reported a small but statistically higher mean CH in NTG eyes than POAG eyes [4]. We are curious about whether there were any differences in corneal biomechanical properties between normal eyes and NTG eyes in this study, as well as any effect of corneal biomechanical properties on IOP measurement in NTG eyes [1].

Z. Chen $(\bowtie) \cdot \mathrm{G} . \mathrm{Xu}$

Eye Department, HuiZhou Municipal Central Hospital,

HuiZhou City, Guangdong Province 516001,

People's Republic of China

e-mail: chenzilin2009@126.com
Another point we would like to mention is that intraocular pressure, just as our blood pressure, has diastolic and systolic pressure [5]. Based on the principle of DCT and ORA, IOP measurement taken by DCT always disaplays as diastolic pressure, while ORA finishes IOP measurement in 20 miniseconds, so it is hard to distinguish whether the IOP measured by ORA is diastolic or systolic pressure. It was speculated that the repeatability of IOP measurement taken by ORA would be not so good as DCT and GAT, which can cause the difference between IOPcc and GAT-IOP and DCT-IOP to a certain extent.

Once again, we appreciate the authors' efforts in investigating IOP measurement with different modalities.

\section{References}

1. Morita T, Shoji N, Kamiya K, Hagishima M, Fujimura F, Shimizu K (2009) Intraocular pressure measured by dynamic contour tonometer and ocular response analyzer in normal tension glaucoma. Graefes Arch Clin Exp Ophthalmol Aug 20 [Epub ahead of print]. doi:10.1007/s00417-009-1169-4

2. The Advanced Glaucoma Intervention Study (AGIS) 7 (2000) The relationship between control of intraocular pressure and visual field deterioration. The AGIS Investigators. Am J Ophthalmol 130:429440

3. Shah S, Laiquzzaman M, Mantry S, Cunliffe I (2008) Ocular response analyser to assess hysteresis and corneal resistance factor in low tension, open angle glaucoma and ocular hypertension. Clin Experiment Ophthalmol 36:508-513

4. Ang GS, Bochmann F, Townend J, Azuara-Blanco A (2008) Corneal biomechanical properties in primary open angle glaucoma and normal tension glaucoma. J Glaucoma 17:259-262

5. Donnelly SJ, Subramanian PS (2009) Relationship of intraocular pulse pressure and spontaneous venous pulsations. Am J Ophthalmol 147(1):51-55 